\title{
ARTICLES
}

\section{NMR Relaxation Study of Tetrapentylammonium Ions in Acetonitrile Solutions}

\author{
E. W. Lang,* S. Bradl, \\ Institut für Biophysik und physik Biochemie, Universität Regensburg, Postfach 397, FRG \\ W. Kunz, and P. Turq \\ Laboratoire d'Electrochimie, Bat. F, Tour 74, Universite Pierre et Marie Curie, 8 rue Cuvier, 75005 Paris, \\ France (Received: April 10, 1991; In Final Form: July 3, 1991)
}

\begin{abstract}
NMR measurements of the spin-lattice relaxation rates of the ${ }^{1} \mathrm{H},{ }^{13} \mathrm{C}$, and ${ }^{14} \mathrm{~N}$ nuclei and spin-echo self-diffusion coefficients are reported for tetrapentylammonium bromide in acetonitrile solutions over a wide range of temperatures and concentrations. The results are discussed in terms of overall and segmental motions of the aliphatic chains of the organic ions. Order parameters characterizing the geometrical constraints upon local segmental motions are given also. Cationic self-diffusion coefficients are found to differ from those obtained with quasi-elastic neutron scattering techniques (QENS). The difference may be explained in terms of fast and slow modes contributing to the diffusion processes and their selective detection with QENS and NMR techniques.
\end{abstract}

\section{Introduction}

The structure and the dynamic properties of a great number of aqueous electrolyte solutions have been thoroughly studied with various experimental methods. The properties of ions in nonaqueous solvents, however, are much less well-known despite their considerable technological and theoretical interest. ${ }^{1}$

The present study is part of a project concerning the behavior of tetraalkylammonium salts dissolved in acetonitrile. Some of these solutions are used in electrochemistry as supporting electrolytes for electrochemical synthesis or analytical techniques. Tetraalkylammonium salts can also serve as phase-transfer catalysts between water and an organic phase.

Tetrapentylammonium bromide solutions are particularly suitable for experimental studies. At room temperature that salt is soluble up to more than $1 \mathrm{M}$ in acetonitrile. The solutions are stable and only slightly hygroscopic. The considerable number of hydrogen atoms ( 44 per molecule) is favorable for neutron scattering experiments. Due to the size of the organic cation it was possible to use small-angle neutron scattering (SANS) techniques to study the static ion-ion correlation functions in deuterated acetonitrile solutions. ${ }^{2}$ The structural results were completed by thermodynamic ${ }^{11}$ measurements yielding osmotic coefficients. Both the SANS and the thermodynamic results could be reproduced by hypernetted-chain integral equation calculations and also by Brownian dynamics simulations. ${ }^{2}$

(1) Barthel, J.; Gores, H. J.; Schmeer, G.; Wachter, R. In Topics in Current Chemistry; Boschke, F. L., Ed.; Springer: Heidelberg, 1983; Vol. 111, pp 33-144.

(2) Kunz, W.; Calmettes, P.; Turq, P. J. Chem. Phys. 1990, 92, 2367.

(3) Kunz, W.: Turq, P.; Bellisent-Funel, M. C.; Calmettes, P. J. Chem. Phys., to be published.

(4) Barthel, J.; Lauermann, G.; Neueder, R. J. Solution Chem. 1986, 15, 51.

(5) Prielmeier, F. X.; Lang, E. W.; Speedy, R. J.; Lüdemann, H.-D. Ber Bunsenges, Phys. Chem. 1988, 92, 1111.

(6) Stilbs, P. Prog. Nucl. Magn. Reson. Spectrosc. 1987, 19, 1.

(7) Weingärtner, M. Z. Phys. Chem. NF 1982, 132, 129.

(8) Krumgalz, B. S. J. Chem. Soc., Faraday Trans. l 1983, 79, 571.

(9) Onsager, L. Ann. N.Y. Acad. Sci. 1945, 46, 241.

(10) Gal. I. J.: Paligoric, I.; Antonijevic, V. G. J. Inorg. Chem. 1970, 32 , 1645

(11) Barthel, J.; Kunz, W. J. Solution Chem. 1988, 17, 399.
Tetrapentylammonium bromide solutions are also suitable for experiments which yield information about the dynamic behavior of ions in solution. Quasi-elastic neutron scattering of the hydrogenated salt in deuterated acetonitrile gives the cation selfdiffusion coefficient. NMR measurements yield complementary information. In fact, the combination of NMR and QENS experiments is particularly informative. This is because of the time-scale separation between the two techniques. NMR uses rather long time scales when compared to QENS where the typical time scale is of the order of picoseconds. Therefore a comparison between NMR and QENS helps to distinguish fast and slow processes in solutions. The QENS results as well as their comparison with the present NMR results will be given in a separate paper. ${ }^{3}$

In this study we present proton and carbon-13 spin-lattice relaxation time and self-diffusion coefficient measurements of the tetrapentylammonium $\left(\mathrm{Pe}_{4} \mathrm{~N}^{+}\right)$cations for a wide range of temperatures and concentrations. The results will be discussed in terms of overall and segmental motions of the different alkyl groups on the aliphatic chains of the organic ions. Characteristic correlation times for these motions will be given as well as order parameters which characterize the geometrical constraints of the local segmental motions. Self-diffusion coefficients of the cations are compared with those obtained from QENS experiments and are found to differ considerably. The difference may be explained in terms of fast and slow modes contributing to the diffusion process and their selective detection with QENS and NMR techniques.

\section{Experiment}

Tetrapentylammonium bromide $\left(\mathrm{Pe}_{4} \mathrm{NBr}\right)$ was purified as indicated in ref 4 and dried on a vacuum line for about $24 \mathrm{~h}$. Deuterated acetonitrile ( 99.6 atom \%) was obtained from the Laboratoire des Molecules Marquees, CEN-Saclay, France, and used without further purification. The solutions were prepared by weighing the proper amount of salt to $3 \mathrm{~g}$ of deuterated solvent. Afterward the solutions were deoxygenated on a vacuum line by at least five freeze-pump-thaw cycles. The sample cell consisted of a precision 5-mm NMR tube (Wilmad, NJ) of length $40 \mathrm{~mm}$ fused to a glass capillary with o.d. $5 \mathrm{~mm}$, i.d. $0.6 \mathrm{~mm}$, and length $120 \mathrm{~mm}$ (Duran 50, Schott, Mainz). The cell was connected to 


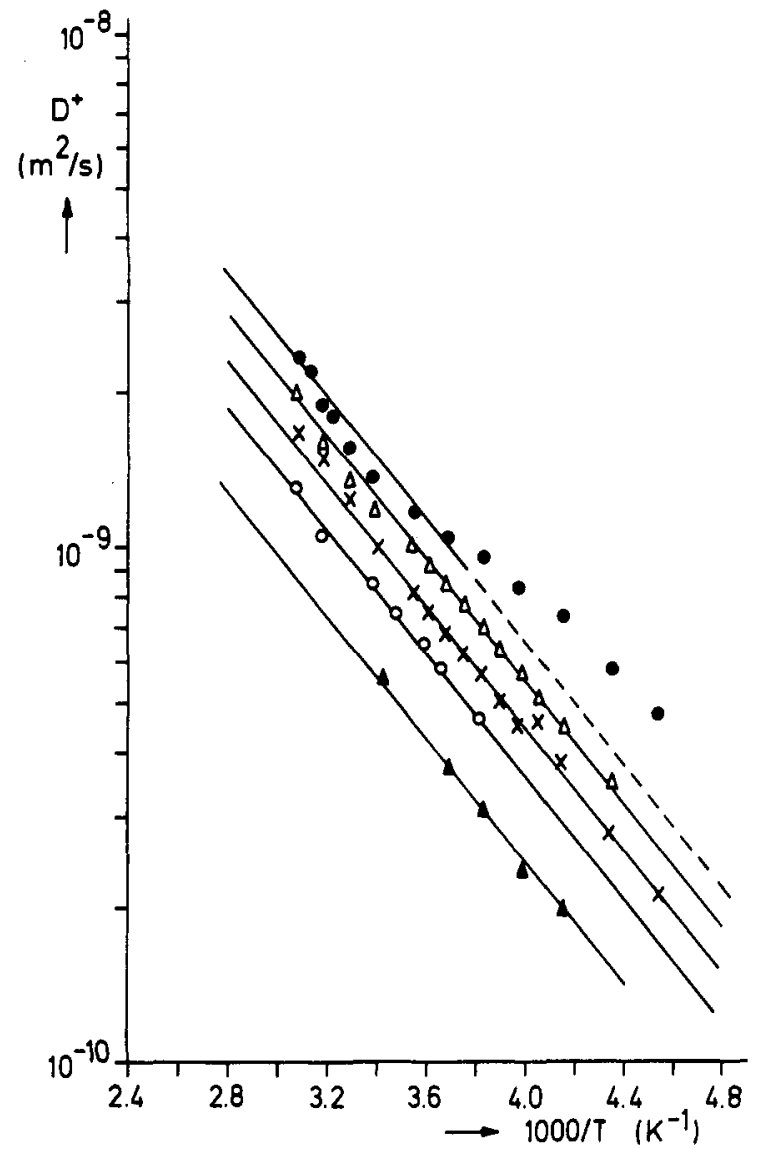

Figure 1. Temperature dependence of the self-diffusion coefficients of the $\mathrm{Pe}_{4} \mathrm{~N}^{+}$cations at various concentrations: $\bullet, 0.01 \mathrm{~m} ; \Delta, 0.1 \mathrm{~m} ; \times 0.2$ $m ; 0,0.5 \mathrm{~m}$, and, $\Delta, 1.0 \mathrm{~m}\left(\mathrm{~m}=\mathrm{mol}\right.$ of salt $/ \mathrm{kg}$ of $\left.\mathrm{CH}_{3} \mathrm{CN}\right)$. Full lines are calculated with eq 2 and the parameters given in Table III.

the vacuum line with a glass cone and was flame-sealed just below the cone after degassing. The glass capillary functioned to minimize convective flow within the cell due to residual temperature gradients along the sample cell.

All experiments were performed on a Bruker MSL 300 NMR spectrometer equipped with a variable-temperature unit VT 1000 and a pulsed magnetic field gradient unit BZ 18B. The gradient unit was modified for a digital setting of the gradient amplitude by a series of fixed resistances. The probe consisted of a home-built 10-mm probe head with two Helmholz coils suitable for operation in the range $280-300 \mathrm{MHz}$. Details of the probe design have been described by Prielmeier et al. ${ }^{5}$ elsewhere. This design assures that the gradient coils are thermally isolated from the sample space so the coils remain at constant temperature during variable-temperature measurements.

For the self-diffusion measurements the Fourier-transform pulsed-gradient spin-echo method (FT-PGSE) was applied to the proton magnetization. ${ }^{6}$ The decay of the spin-echo amplitude is given by

$A(2 \tau)=A(0) \exp \left(-2 \tau / T_{2}\right) \exp \left(-\left(\gamma_{\mathrm{H}} g \delta\right)^{2}\left(t_{\mathrm{p}}-\delta / 3\right) D\right)$

$T_{2}$ represents the proton spin-spin relaxation time, $\gamma_{H}$ is the gyromagnetic ratio of the proton, $g=k I$ is the field gradient strength with $I$ the gradient coil current, $\delta$ gives the length of the gradient pulse, $t_{\mathrm{p}}$ is the gradient pulse spacing, and $D$ is the self-diffusion coefficient. During the experiments the delay time between the $90^{\circ}$ and the $180^{\circ}$ pulses was kept constant, typically between 60 and $150 \mathrm{~ms}$, and $t_{\mathrm{p}}=\tau$ was used throughout. The amplitude of the field gradient pulse $g=k I$ was varied by increasing the coil current digitally. The applied gradient strength typically ranged from $9.6 \times 10^{-3} \mathrm{~T} / \mathrm{m}$ to $37.1 \times 10^{-3} \mathrm{~T} / \mathrm{m}$. The coil constant $k$ was calibrated with a sample of water with a precisely known self-diffusion coefficient. ${ }^{7}$ The amplitude of the methyl proton peak was sampled with the $T_{1}$ peak picking routine

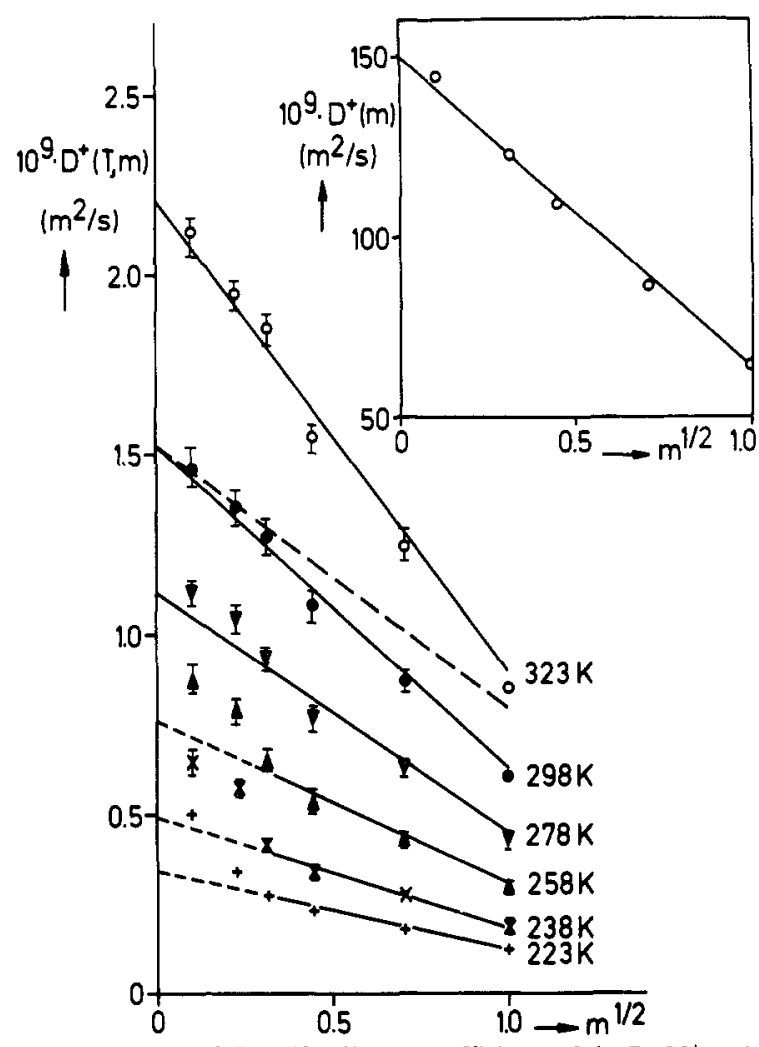

Figure 2. Isotherms of the self-diffusion coefficients of the $\mathrm{Pe}_{4} \mathrm{~N}^{+}$cations as a function of the square root of the concentration $m$ (molality scale). The insert shows the preexponential factors $D^{+}(m)$ of eq 2 as tabulated in Table III.

for 10-15 different field gradient settings. The delay time $\tau$ was adjusted to minimize the echo amplitudes of the neighboring methylene proton resonances due to $J$-modulation effects. ${ }^{6} \mathrm{Be}$ cause the time delay between the $90^{\circ}$ and $180^{\circ}$ pulses was kept constant in each experiment the spin-spin relaxation and $J$ modulation effects were equal for each Fourier transform of the second half of the corresponding spin-echo signal. The time scale during which molecular diffusion is monitored is given in the PGSE experiment by $\left(t_{\mathrm{p}}-\delta / 3\right)$ and ranged from 50 to $70 \mathrm{~ms}$. The standard deviation of the fitted diffusion coefficients was always better than $2 \%$. Reproducibility considerations, however, indicate that the overall accuracy is $\pm 5 \%$. Spin-lattice relaxation times $T_{1}$ were determined with the inversion-recovery pulse sequence $\left(180^{\circ}-\tau-90^{\circ}\right)$ with alternating phases and the ${ }^{13} \mathrm{C} T_{1}$ were measured during broad-band proton decoupling. Data were analyzed with a three-parameter nonlinear least-squares regression program. The relaxation times are considered reliable to $\pm 10 \%$. The temperature was varied with a flow of cold nitrogen gas appropriately heated and controlled with the VT 1000 unit. It was measured at the center of the rf coil with a chromel-alumel thermocouple before and after each series of spin-echo experiments. The temperatures are considered reliable to $\pm 1 \mathrm{~K}$.

Self-Diffusion Coefficients of the $\mathrm{Pe}_{4} \mathrm{~N}^{+}$Cations. Figure 1 presents the self-diffusion coefficients $D^{+}$of the $\left(\mathrm{Pe}_{4} \mathrm{~N}\right)^{+}$cations, at the concentrations indicated. The temperature dependence follows a simple Arrhenius law given by $\left(m=\mathrm{mol}\right.$ of $\mathrm{Pe}_{4} \mathrm{~N}^{+} / \mathrm{kg}$ of $\mathrm{CD}_{3} \mathrm{CN}$ )

$$
D^{+}(T, m)=D^{+}(m) \exp \left(E_{\mathrm{a}} / R T\right)
$$

The parameters $D^{+}(m)$ and $E_{\mathrm{a}}$ are compiled in Table III. The apparent activation energy $E_{\mathrm{a}}=11.4 \mathrm{~kJ} / \mathrm{mol}$ is within the scatter of the experimental data identical for all concentrations except for the most dilute solutions $(0.01 \mathrm{~m}$ and $0.05 \mathrm{~m})$ at low temperatures where a slope corresponding to $E_{\mathrm{a}}=7.6 \mathrm{~kJ} / \mathrm{mol}$ is obtained.

Figure 2 illustrates the dependence of the cationic self-diffusion coefficients $D^{+}$on the solute concentration $m$ for some representative isotherms. The data have been deduced from Figure 


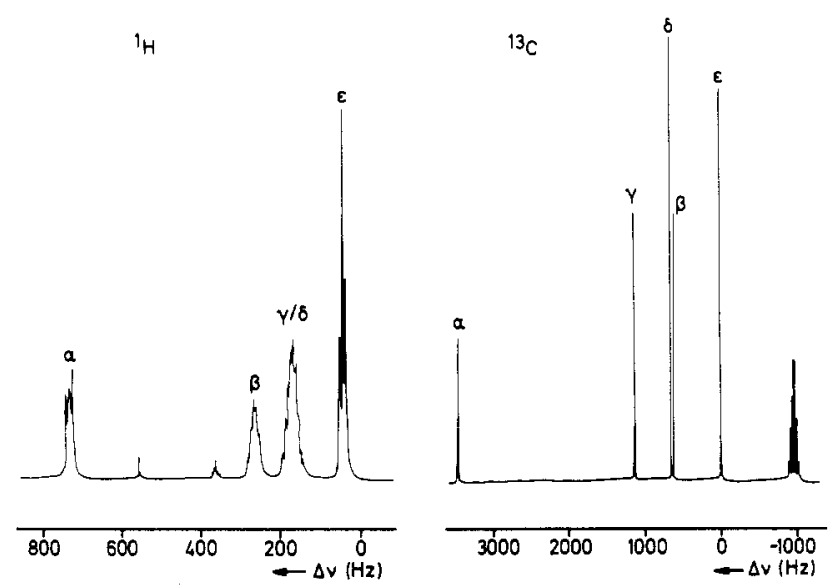

Figure 3. Proton and carbon-13 spectrum of a $0.5 \mathrm{~m} \mathrm{Pe} \mathrm{NBr}_{4} / \mathrm{CD}_{3} \mathrm{CN}$ solution. The resonances $\alpha$ to $\epsilon$ correspond to the alkyl groups of the organic cations. $\alpha$ designates the methylene groups closest to the central nitrogen and $\epsilon$ designates the terminal methyl groups. The proton resonances corresponding to the $\gamma$-methylene and $\delta$-methylene groups could not be resolved in a field of $7 \mathrm{~T}(300 \mathrm{MHz})$. The assignment of the carbon- 13 peaks follows the order of their spin-lattice relaxation times as discussed in the text.

1 by interpolation. The $D^{+}$depend linearly on $m^{1 / 2}$ for all but the low-temperature data of the most dilute solution. This suggests a simple representation of the preexponential factor $D^{+}(m)$ in eq 2 according to (see insert Figure 2)

$$
D^{+}(m)=D^{+}(0)-A m^{1 / 2}
$$

with

$$
D^{+}(0)=(1.50 \pm 0.08) \times 10^{-7} \mathrm{~m}^{2} / \mathrm{s}
$$

and

$$
A=(0.86 \pm 0.1) \times 10^{-7}\left(\mathrm{~m}^{2} \mathrm{~kg}^{1 / 2}\right) /\left(\mathrm{mol}^{1 / 2} \mathrm{~s}\right)
$$

At $298 \mathrm{~K}$ the limiting cationic self-diffusion coefficient may be obtained by extrapolation, yielding

$$
D^{+}(T=298 \mathrm{~K}, m=0)=(1.52 \pm 0.06) \times 10^{-9} \mathrm{~m}^{2} / \mathrm{s}
$$

Krumgalz ${ }^{8}$ predicted $1.49 \times 10^{-9} \mathrm{~m}^{2} / \mathrm{s}$ by means of a separation of limiting equivalent conductances. For comparison Onsager's limiting law ${ }^{9}$ is also given in Figure 2 for the $298 \mathrm{~K}$ isotherm.

Diffusion coefficients lower than those calculated by the limiting law normally indicate a high cation-anion association as has indeed been observed in nonpolar solvents by osmotic measurements. ${ }^{10}$ For the solution under consideration the association constant is, however, quite small. ${ }^{11}$ This result is also in accord with NMR chemical shift measurements which demonstrate decreasing association with increasing length of the alkyl chains in quaternary ammonium salts dissolved in acetonitrile. ${ }^{12}$ Hence, the rapid decrease of the cation self-diffusion coefficient $D^{+}$as a function of concentration must be due to other effects.

In a parallel study ${ }^{3} \mathrm{QENS}$ experiments have been performed on a $0.43 \mathrm{M} \mathrm{Pe}_{4} \mathrm{NBr} / \mathrm{CD}_{3} \mathrm{CN}$ solution. Diffusion coefficients of the cations extracted from a fit to the dynamic structure factor $S(q)$ are significantly higher than those obtained from NMR experiments. The difference may be explained by the different time scales covered by QENS $(\approx$ picosecond $)$ and NMR $(\approx$ millisecond). Two effects may be expected to contribute to ionic diffusion in liquids of low viscosity:

(a) A second-order hydrodynamic effect acting in the picosecond time scale is the first effect. It is approximately proportional to the salt concentration, the difference between the self-diffusion coefficients of cation and anion, and also inversely proportional

(12) Kabisch, G. Ber. Bunsenges. Phys. Chem. 1976, 80, 602

(13) Kunz, W.; Turq, P.; Bellissent-Funel, M. C.; Calmettes, P.; Kahn, K. unpublished results.
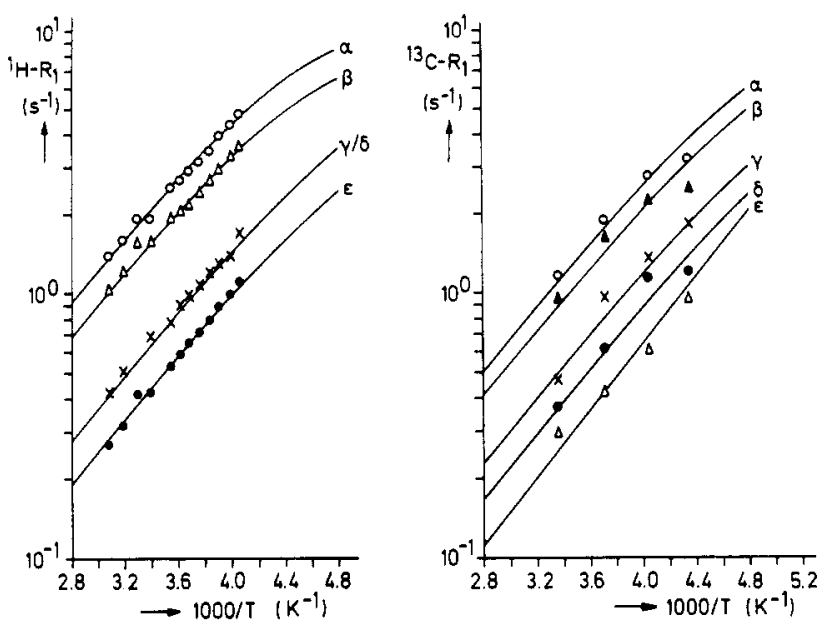

Figure 4. Proton and carbon-13 spin-lattice relaxation rates in a $0.1 \mathrm{~m}$ $\mathrm{Pe}_{4} \mathrm{NBr} / \mathrm{CD}_{3} \mathrm{CN}$ solution of the alkyl group resonances as assigned in Figure 3. The full lines are calculated with eqs 14,18 , and $19\left({ }^{1} \mathrm{H}\right)$ and eq $5\left({ }^{13} \mathrm{C}\right)$ and the parameters given in Table $\mathrm{V}$ and $\mathrm{IV}$, respectively.
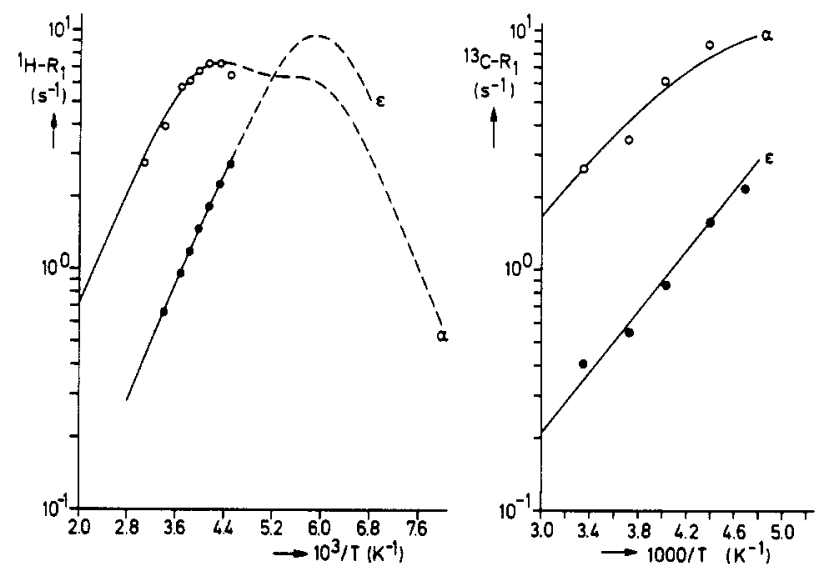

Figure 5. Proton and carbon-13 spin-lattice relaxation rates in a $1.0 \mathrm{~m}$ $\mathrm{Pe}_{4} \mathrm{NBr} / \mathrm{CD}_{3} \mathrm{CN}$ solution. Only the central $\alpha$-methylene and the terminal methyl group relaxation rates are shown to avoid data overlap. Full lines are calculated according to eqs 14,18 , and $19\left({ }^{1} \mathrm{H}\right)$ and eq 5 $\left({ }^{13} \mathrm{C}\right)$ with the parameters given in Tables $\mathrm{V}$ and IV, respectively.

to the square of the solution viscosity. ${ }^{14}$ In solvents of low viscosity it may cause a noticeable contribution to ionic diffusion processes.

(b) For longer time scales a relaxation effect is expected to dominate. It is essentially the time ions need to follow a path leading to significant charge separation and back to an equilibrium configuration. ${ }^{15}$ These arrangements typically take place on the order of nanoseconds, a time scale not covered by QENS but covered by NMR measurements.

Consequently the difference between $D^{+}(\mathrm{NMR})$ and $D^{+}$. (QENS) is supposed to come from a relaxation effect essentially. This could indeed be corroborated by Brownian dynamics calculations of the relaxation contribution to ionic diffusion. ${ }^{3}$

${ }^{1} \mathrm{H}$ and ${ }^{13} \mathrm{C}$ Spin-Lattice Relaxation Rates of $\mathrm{Pe}_{4} \mathrm{~N}^{+}$Ions. Proton spin-lattice relaxation rates of the cations have been measured over a large concentration and temperature range. The experimental data are tabulated in Table I. Figure 3 shows the assignment of the proton resonances to the different alkyl groups $\left(\mathrm{CH}_{n}\right)$ of the cation with $\mathrm{C}_{\alpha} \mathrm{H}_{2}$ the methylene group nearest to the central nitrogen atom and $\mathrm{C}_{\mathrm{k}} \mathrm{H}_{3}$ the methyl group of the alkyl chain. It may be seen that the proton resonances of the $\mathrm{C}_{\gamma} \mathrm{H}_{2}$ and $\mathrm{C}_{\delta} \mathrm{H}_{2}$ methylene groups overlap strongly, hence they could not be resolved. Only an average relaxation rate can be given in Table I therefore. Figures 4 and 5 show the temperature dependence of the proton relaxation rates for the $0.1 \mathrm{~m}$ and the $1 \mathrm{~m}$ solutions.

(14) Turq, P.; Brun, B.; Chemla, M. J. Chim. Phys. 1973, 70, 661.

(15) Zhong, E. C.; Friedman, H. L. J. Phys. Chem. 1988, 92, 1685. 
TABLE I: Proton Spin-Lattice Relaxation Times $T_{1}$ (ms) of the Alkyl Groups $\left(\mathrm{C}_{i} \mathrm{H}_{m} i=\alpha, \ldots, \epsilon\right)$ of the $\mathrm{Pe}_{4} \mathrm{~N}^{+}$Cations $^{a}$

\begin{tabular}{rrrrr}
\hline & \multicolumn{4}{c}{$\mathrm{H} / T_{1}, \mathrm{~ms}$} \\
\cline { 2 - 5 }$T, \mathrm{~K}$ & $\mathrm{C}_{\alpha}$ & $\mathrm{C}_{\beta}$ & $\mathrm{C}_{8 / \delta}$ & $\mathrm{C}_{\varepsilon}$ \\
\hline & & $0.1 \mathrm{~m}$ & & \\
325.0 & 719 & 971 & 2370 & 3720 \\
314.0 & 626 & 827 & 1970 & 3160 \\
304.0 & 517 & 647 & 1380 & 2400 \\
295.0 & 519 & 638 & 1460 & 2361 \\
282.5 & 396 & 520 & 1290 & 1880 \\
277.0 & 372 & 490 & 1110 & 1700 \\
272.0 & 342 & 465 & 1020 & 1540 \\
266.5 & 315 & 416 & 928 & 1410 \\
261.0 & 287 & 375 & 836 & 1260 \\
256.5 & 252 & 341 & 773 & 1130 \\
251.0 & 229 & 305 & 722 & 1010 \\
247.0 & 208 & 277 & 593 & 907 \\
& & $0.2 m$ & & \\
324.0 & 651 & 882 & 2180 & 3590 \\
314.0 & 558 & 739 & 1740 & 3000 \\
304.0 & 478 & 652 & 1600 & 2560 \\
293.5 & 404 & 532 & 1300 & 2090 \\
282.0 & 335 & 455 & 1060 & 1710 \\
277.0 & 306 & 414 & 961 & 1550 \\
272.0 & 281 & 381 & 848 & 1390 \\
267.0 & 256 & 348 & 792 & 1250 \\
262.0 & 245 & 332 & 776 & 1160 \\
257.0 & 225 & 296 & 666 & 1020 \\
252.0 & 203 & 275 & 691 & 935 \\
247.0 & 183 & 244 & 553 & 840 \\
241.5 & 180 & 240 & 503 & 767 \\
230.5 & 146 & 196 & 478 & 584 \\
& & $0.5 m$ & & \\
325.0 & 493 & 676 & 1780 & 3090 \\
314.0 & 412 & 564 & 1470 & 2610 \\
295.0 & 337 & 445 & 1090 & 1890 \\
287.0 & 290 & 374 & 841 & 1630 \\
272.0 & 221 & 280 & 710 & 1170 \\
262.0 & 199 & 274 & 593 & 965 \\
& & $1.0 m$ & & \\
324.0 & 361 & & & \\
314.0 & & & 1054 & \\
292.0 & 255 & 307 & 820 & 1510 \\
271.0 & 174 & 242 & 617 & 1050 \\
261.0 & 164 & 213 & 535 & 854 \\
251.0 & 149 & 188 & 369 & 684 \\
241.0 & 139 & 173 & 332 & 553 \\
231.0 & 139 & 170 & 288 & 446 \\
222.0 & 156 & 184 & 270 & 369 \\
& & & &
\end{tabular}

${ }^{a}$ Carbon atoms are enumerated in ascending order along the aliphatic chain with the $\alpha$-carbon closest to the central nitrogen. The resonances of the $\mathrm{C}_{\delta} \mathrm{H}_{n}$ and $\mathrm{C}_{\delta} \mathrm{H}_{n}$ groups could not be resolved. Solute concentrations are given on a molality scale $(m=\mathrm{mol}$ of salt $/ \mathrm{kg}$ of $\mathrm{CH}_{3} \mathrm{CN}$ ).

${ }^{13} \mathrm{C}$ spin-lattice relaxation rates have been determined in addition for the $0.1 \mathrm{~m}, 0.5 \mathrm{~m}$, and $1.0 \mathrm{~m}$ solutions over the same $T, p$ range. The experimental data are tabulated in Table II. The ${ }^{13} \mathrm{C}$ spectrum is simple with the resonances from the different alkyl groups $\left(\mathrm{CH}_{2}\right)$ well resolved (see Figure 3 ). The various ${ }^{13} \mathrm{C}$ peaks have been assigned to the different alkyl groups according to their spin-lattice relaxation rates assuming a monotonic increase of the mobility of the alkyl groups with increasing distance to the nitrogen core. ${ }^{16}$ The relaxation rates at ambient temperatures agree very well with data reported in the literature. ${ }^{16}$

These relaxation rates exhibit a number of trends. As expected, they decrease along the alkyl chain for a given concentration, reflecting a regular increase in the segmental motion from the constrained end $\left(\mathrm{C}_{\alpha} \mathrm{H}_{2}\right)$ toward the highly mobile methyl groups $\left(\mathrm{C}_{\mathrm{f}} \mathrm{H}_{3}\right)$. Increasing the solute concentration at a given temperature affects the relaxation rates of the various alkyl groups differently. 29.

(16) Dando, N. R.; Gold, H. S.; Dybowski, C. Appl. Spectrosc. 1983, 37,
TABLE II: Carbon-13 Spin-Lattice Relaxation Times $T_{1}$ (ms) of the Alkyl Groups $\left(\mathrm{C}_{i} \mathrm{H}_{n}, i=\alpha, \ldots, \epsilon\right)$ of the $\mathrm{Pe}_{4} \mathrm{~N}^{+}$Cations ${ }^{a}$

\begin{tabular}{|c|c|c|c|c|c|}
\hline \multirow[b]{2}{*}{$T, \mathrm{~K}$} & \multicolumn{5}{|c|}{${ }^{13} \mathrm{C} / T_{1}, \mathrm{~ms}$} \\
\hline & $\mathrm{C}_{\alpha}$ & $C_{\beta}$ & $\mathrm{C}_{\hat{\delta}}$ & $\mathrm{C}_{\delta}$ & $\mathrm{C}_{\mathrm{e}}$ \\
\hline \multicolumn{6}{|c|}{$0.1 \mathrm{~m}$} \\
\hline 297.0 & 865 & 1060 & 2160 & 2720 & 3380 \\
\hline 269.0 & 531 & 623 & 1050 & 1630 & 2360 \\
\hline 247.0 & 362 & 449 & 741 & 884 & 1630 \\
\hline 230.0 & 311 & 401 & 550 & 837 & 1050 \\
\hline \multicolumn{6}{|c|}{$0.5 \mathrm{~m}$} \\
\hline 323.0 & 947 & 1340 & 2890 & 3880 & 5420 \\
\hline 297.0 & 614 & 793 & 1430 & 2340 & 3320 \\
\hline 268.0 & 398 & 544 & 959 & 1440 & 2150 \\
\hline 247.0 & 248 & 298 & 531 & 757 & 1180 \\
\hline 230.0 & 213 & 238 & 373 & 597 & 859 \\
\hline 220.0 & 177 & 203 & 358 & 563 & 875 \\
\hline \multicolumn{6}{|c|}{$1.0 \mathrm{~m}$} \\
\hline 298.0 & 381 & 477 & 972 & 1480 & 2461 \\
\hline 268.0 & 288 & 340 & 624 & 1070 & 1820 \\
\hline 248.0 & 163 & 218 & 404 & 700 & 1160 \\
\hline 227.0 & 114 & 133 & 222 & 347 & 629 \\
\hline 213.0 & & & & & 456 \\
\hline
\end{tabular}

${ }^{a}$ Indices correspond to those given in Table I,

TABLE III: Apparent Activation Energy $E$, and Preexponential Factor $D^{+}(m)$ Appropriate To Represent the Temperature Dependence of the Self-Diffusion Coefficients of $\mathrm{Pe}_{4} \mathrm{~N}^{+}$According to Eq 2

\begin{tabular}{lll}
\hline$D^{+}(\mathrm{m}), \mathrm{m} / \mathrm{s}^{2}$ & $E_{\mathrm{a}}, \mathrm{kJ} / \mathrm{mol}$ & $c, m$ \\
\hline $1.50 \times 10^{-7}$ & $11.4 / 7.6$ & 0.01 \\
$1.38 \times 10^{-7}$ & $11.4 / 7.6$ & 0.05 \\
$1.30 \times 10^{-7}$ & 11.4 & 0.10 \\
$1.06 \times 10^{-7}$ & 11.4 & 0.20 \\
$8.56 \times 10^{-8}$ & 11.4 & 0.50 \\
$5.85 \times 10^{-8}$ & 11.4 & 1.00
\end{tabular}

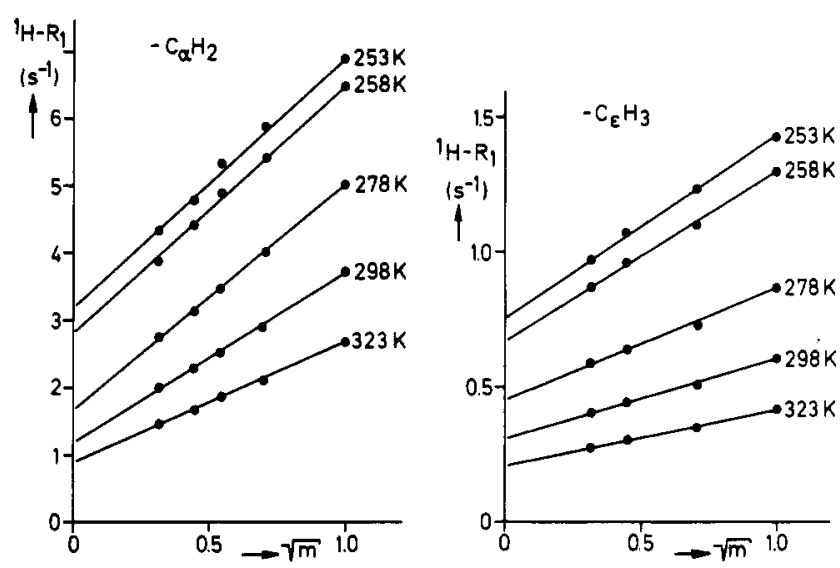

Figure 6. Isothermal concentration dependence of the proton relaxation rates of the $\alpha$-methylene and the terminal $\epsilon$-methyl group resonances of the $\mathrm{Pe}_{4} \mathrm{~N}^{+}$cations. Note the different scale of the $y$-axis in both figures.

The rate of the terminal methyl groups of the $\mathrm{Pe}_{4} \mathrm{~N}^{+}$cations exhibits comparatively little dependence upon salt concentration (Figure 6). But this dependence becomes increasingly pronounced with decreased distance from the constrained end of the alkyl chain at the nitrogen center. The relaxation rates of the $\alpha$-methylene groups then exhibit a strong concentration dependence, which has been shown to parallel the concentration dependent of the bulk solution viscosity. ${ }^{17}$ The latter increases strongly in concentrated solution causing a strong slowing down of overall molecular tumbling motions of the dissolved cations. Because of it, the proton relaxation rate of the $\alpha$-methylene groups closest to the nitrogen center was found to have a maximum at low temperatures in the $1.0 \mathrm{~m}$ solution. At the maximum the correlation time for the

(17) Dando, N. R.; Gold, H. S.; Dybowski, C. Org. Magn. Reson. 1983, $21,467$. 
overall tumbling motion of the cations becomes comparable to the inverse of the Larmor frequency of the protons. The latter is roughly four times larger than the corresponding ${ }^{13} \mathrm{C}$ Larmor frequency. Hence the equivalent maximum in the ${ }^{13} \mathrm{C}$ relaxation rate should be detected at lower temperatures only. However, a freezing transition intervenes and prohibits its experimental observation.

Evaluation of the ${ }^{13} \mathrm{C}$ Relaxation Rates. The spin-lattice relaxation of the carbon-13 nuclei in $\mathrm{Pe}_{4} \mathrm{~N}^{+}$ions is mainly due to fluctuating dipolar interactions with directly bonded protons. The dipolar interaction is represented by a second-rank tensor, the components of which are generally known in a molecule-fixed principal axis system (PAS). The tensor is axially symmetric about the $\mathrm{C}-\mathrm{H}$ bond of an alkyl group. The orientation of the PAS relative to the laboratory axis system (LAB) may be given with second-rank Wigner rotation matrices $\mathbf{D}_{\mathrm{mn}}\left(\Omega_{\mathrm{LP}}\right)$. Their components are time dependent because of molecular reorientations. The latter may be decomposed into isotropic overall tumbling motions and anisotropic segmental motions within the aliphatic chains. Both motional modes may be assumed to be statistically independent. Conformation transitions of the aliphatic chains have been treated in the literature with varying degrees of rigor. ${ }^{18}$

Dipolar spin-lattice relaxation rates of carbon-13 nuclei due to a coupling to $n$ directly bonded protons are given by ${ }^{19}$

$$
\begin{aligned}
& R_{10 \mathrm{R}}\left({ }^{13} \mathrm{C}-{ }^{1} \mathrm{H}\right)=n(2 / 15)\left(\gamma_{\mathrm{H}} \gamma_{\mathrm{C}} \hbar \mu_{0} / 4 \pi\right)^{2} S(S+1) \times \\
&\left(g^{(0)}\left(\omega_{\mathrm{C}}-\omega_{\mathrm{H}}\right)+3 g^{(1)}\left(\omega_{\mathrm{C}}\right)+6 g^{(2)}\left(\omega_{\mathrm{C}}+\omega_{\mathrm{H}}\right)\right)
\end{aligned}
$$

with $\gamma_{\mathrm{H}}, \gamma_{\mathrm{C}}$ the gyromagnetic ratios of the protons and carbon-13 nuclei, respectively, and $S=1 / 2$ the spin of the protons. The other symbols have their usual meaning. The spectral density $g(\omega)$ is the Fourier transform of the correlation function $G(t)$ for the Wigner component $D_{0 \mathrm{q}}\left(\Omega_{L P}\right)$, which relates the local interaction frame (PAS) to the laboratory frame (LAB). This transformation is first performed to a diffusion frame $D$ of the nitrogen core and then to the local interaction frame $P$. If the overall tumbling motion is assumed to be uncoupled from the segmental motions, the correlation function may be written as ${ }^{18}$

$$
G_{\mathrm{OR}}(t)=G_{\mathrm{rot}}(t) G_{\mathrm{int}}(t)
$$

As a further simplification the overall tumbling motion will be represented by an isotropic rotational diffusion process ${ }^{19}$

$$
G_{\text {rot }}(t)=(1 / 5) \exp \left(-t / \tau_{\mathrm{or}}\right)
$$

with the tumbling correlation time $\tau_{\text {or }}=1 /\left(6 D_{\text {rot }}\right)$ related to the rotational diffusion coefficient $D_{\text {rot }}$ in the usual way.

Segmental motions may be represented by a random walk between sites identified with the stable conformers of an aliphatic chain. A complete calculation of the specific correlation functions which describe the time evolution of the angular functions probed with ${ }^{13} \mathrm{C}$ NMR has been given by Moro et al. ${ }^{20-22}$ These authors showed that the correlation function $G_{\mathrm{OR}}(t)$ for the $n$th alkyl group $\left(\mathrm{C}_{n} \mathrm{H}_{2}\right)$ on an aliphatic chain can be written in a form equivalent to the "model-free" approach of Lipari and $\mathrm{Sza}^{\prime 23} \mathrm{O}^{23}$ or to the two-step model of Halle and Wennerström ${ }^{24}$ as

$$
G_{\mathrm{OR}}(t)=G_{\mathrm{rot}}(t)\left\{\left(S_{n}\right)^{2}+\left(1-\left(S_{n}\right)^{2}\right) G_{n}(t)\right\}\left\langle\left(1 / R_{\mathrm{CH}}\right)^{3}\right)^{2}
$$

with $R_{\mathrm{CH}}=0.109 \mathrm{~nm}$ the $\mathrm{CH}$ bond length within an alkyl group. The order parameter $\left(S_{n}\right)^{2}$ is a measure of the degree of spatial restriction of the segmental motions. It vanishes in the case of

(18) Moro, G. J.; Ferrarini, A.; Polimeno, A.; Nordio, P. L. In Reactive and Flexibile Molecules in Liquids; Dorfmüller, Th., Ed; Kluwer: Dordrecht, 1989; pp 107-139.

(19) Abragam, A. The Principles of Nuclear Magnetism; Oxford University Press: Oxford, 1961.

(20) Ferrarini, A.; Moro, G.; Nordio, P. L. Mol. Phys. 1988, 63, 225.

(21) Coletta, F.; Moro, G.; Nordio, P. L. Mol. Phys, 1987, 61, 1259.

(22) Coletta, F.; Ferrarini, A.; Nordio, P. L. Chem. Phys. 1988, I23, 397

(23) Lipari, G.; Szabo, A. J. Am. Chem. Soc. 1982, 104, 4546.

\begin{tabular}{|c|c|c|c|c|c|c|c|c|}
\hline$\tau_{0}$, fs & $\begin{array}{c}E_{\mathrm{a}}, \\
\mathrm{kJ} / \mathrm{mol}\end{array}$ & $R_{\mathrm{CH}}, \mathrm{nm}$ & $\left(S_{\alpha}\right)^{2}$ & $\left(S_{\beta}\right)^{2}$ & $\left(S_{\gamma}\right)^{2}$ & $\left(S_{\delta}\right)^{2}$ & $\left(S_{t}\right)^{2}$ & $c, m$ \\
\hline 42.5 & 12.25 & 0.109 & 0.77 & 0.67 & 0.45 & 0.35 & 0.00 & 1.0 \\
\hline 36.0 & 12.25 & 0.109 & 0.70 & 0.60 & 0.42 & 0.25 & 0.00 & 0.5 \\
\hline 30.0 & 12.25 & 0.109 & 0.70 & 0.62 & 0.42 & 0.32 & 0.00 & 0.1 \\
\hline
\end{tabular}

(24) Halle, B.; Wennerström, H, J. Chem. Phys. 1981, 75, 1928.
TABLE IV: Parameters Representing the Carbon-13 Spin-Lattice Relaxation Rates $R_{1}\left({ }^{13} \mathrm{C}\right)$ of the $\mathrm{Pe}_{4} \mathrm{~N}^{+}$Cations According to $\mathrm{Eq} 5 \mathrm{As}$ Discussed in the Text

a completely isotropic internal motion and equals one in case of a totally restricted segmental motion. The corresponding spectral density functions $g_{O R}(\omega)$ are given by

$g_{\mathrm{OR}}(\omega)=$

$$
\left(S_{n}\right)^{2}\left(\tau_{\mathrm{OR}} /\left(1+\left(\omega \tau_{\mathrm{or}}\right)^{2}\right)\right)+\left(1-\left(S_{n}\right)^{2}\right)\left(\tau /\left(1+(\omega \tau)^{2}\right)\right)
$$

where

$$
1 / \tau=1 / \tau_{\mathrm{OR}}+1 / \tau_{n}
$$

In the fast motions limit $(\tau \ll 1)$ eq 9 reduces to

$$
g_{\mathrm{OR}}(\omega)=\left(S_{n}\right)^{2} \tau_{\mathrm{OR}}+\left(1-\left(S_{n}\right)^{2}\right) \tau
$$

No assumption concerning relative time scales of overall tumbling motion and local segmental motion has to be made in eq 8 , though time scale separation would provide a strong justification for the assumption of uncoupled motional modes. ${ }^{24,25}$ To obtain information about segmental motions the overall tumbling correlation time $\tau_{\text {or }}(\mathrm{T})$ has to be known. We will make the assumption that in view of the large size of the cations $\mathrm{Pe}_{4} \mathrm{~N}^{+}$the DebyeStokes-Einstein relation ${ }^{26}$ with sticky boundary conditions may be used, yielding

$$
\tau_{\mathrm{OR}}=(2 / 9)\left(\left(R_{+}\right)^{2} / D^{+}\right)
$$

$R_{+}=0.529 \mathrm{~nm}$ is the radius of the cations and $D^{+}$is their translational diffusion coefficient. It is assumed to be given by the experimental self-diffusion coefficient of the protons of $\mathrm{Pe}_{4} \mathrm{~N}^{+}$. An isotropic overall reorientation of the $\mathrm{Pe}_{4} \mathrm{~N}^{+}$ions is certainly a somewhat crude approximation as the ion is more nearly oblate than spherical. An anisotropic diffusion model with two different components of the diffusion tensor would seem more appropriate. This complication has not been pursued, further, however, to keep the number of adjustable parameters as small as possible.

The local reorientational motion of the methyl groups of the aliphatic chains is assumed not to be restricted spatially, hence $\left(S_{\epsilon}\right)^{2}\left({ }^{13} \mathrm{C}\right)=0$ has been set. The methyl group ${ }^{13} \mathrm{C}$ relaxation rate is then determined predominantly by the fast local reorientational motion of the $\left(\mathrm{C}_{6} \mathrm{H}_{3}\right)$ group around the $\mathrm{C}_{\delta}-\mathrm{C}_{6}$ bond according to eqs 9 and 10 , hence time-scale separation pertains. The corresponding correlation time $\tau_{\epsilon}$ follows an Arrhenius dependence with

$$
\tau_{\epsilon}=\tau_{0} \exp \left(E_{\mathrm{a}} / k T\right)
$$

The parameters $\tau_{0}$ and $E_{\mathrm{a}}$ may then be extracted from the experimental relaxation rates $R_{1}\left({ }^{13} \mathrm{C}_{\mathrm{f}}\right)$ directly. The high-temperature slope of the experimental relaxation rates $R_{1}\left({ }^{13} \mathrm{C}_{n}\right)$ corresponding to the methylene groups on the aliphatic chains is hardly different from the slope of the methyl group relaxation rates $R_{1}\left({ }^{13} \mathrm{C}_{\epsilon}\right)$. Hence, the temperature dependence of the correlation times $\tau_{n}$ corresponding to the $n$th segmental motion have been assumed to follow the Arrhenius form with $\tau_{0}$ and $E_{\mathrm{a}}$ kept independent of the position of the alkyl group on the chain. Thus only the generalized order parameter $\left(S_{n}\right)^{2}$ had to be adjusted for each alkyl group to reproduce the experimental ${ }^{13} \mathrm{C}$ relaxation rates $R_{1}\left({ }^{13} \mathrm{C}_{n}\right)$. These $\left(S_{n}\left({ }^{13} \mathrm{C}_{n}\right)\right)^{2}$ values are listed in Table IV together with the parameters $\tau_{0}$ and $E_{\mathrm{a}}$. The order parameters $\left(\mathrm{S}_{n}\left({ }^{13} \mathrm{C}\right)\right)^{2}$ increase regularly from the highly mobile methyl group toward the $\alpha$-methylene group. The latter is highly constrained due to the large hydrodynamic friction involved in any conformational transition encompassing the motion of a long tail in a viscous medium. The order parameters are in qualitative

(25) Halle, B.; Wennerström, H.; Piculell, L. J. Phys. Chem. 1984, 88, 2482

(26) Kivelson, D. Discuss, Faraday Soc. 1977, 11, 7 
TABLE V: Parameters Representing the Proton Spin-Lattice Relaxation Rates $R_{1}\left({ }^{1} \mathrm{H}\right)$ of the $\mathrm{Pe}_{4} \mathrm{~N}^{+}$Cations According to Eqs 14, 18, and 19 As Discussed in the Text

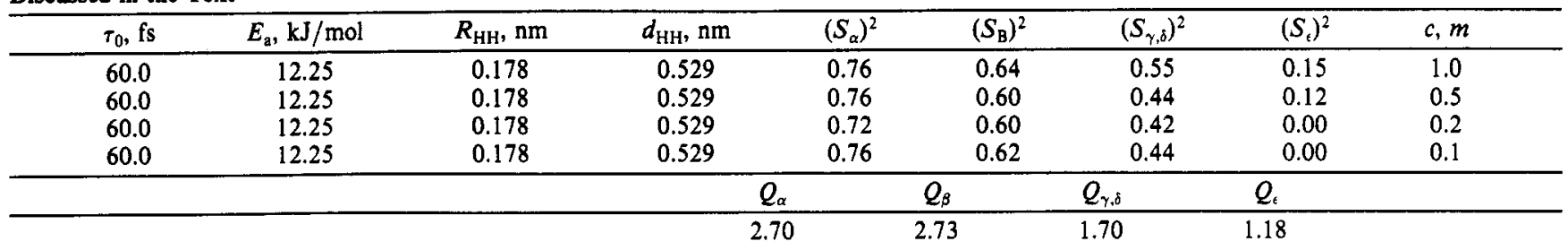

agreement with calculations presented by Ferrarini et al. ${ }^{20}$ for a relatively large value of the trans-gauche transition barrier, hence a high trans population of the alkyl chains. According to eq 9 a large order parameter $\left(S_{n}\right)^{2}$ leaves a large weight to the contribution from overall tumbling motions to the total relaxation rate. This is in accord with the strong concentration dependence of the $\alpha$-methylene carbon relaxation rate, which stems mainly from the viscosity of the solution.

Evaluation of ' $\mathbf{H}$ Relaxation Rates $\boldsymbol{R}_{1}\left({ }^{1} \mathbf{H}\right)$. Proton spin-lattice relaxation rates may be decomposed generally into a sum of intraand intermolecular contributions. The intermolecular contribution $R_{1, \text { Pos }}$ is due to dipolar interactions of the protons on different molecules. As the solvent is fully deuterated only the dipolar interaction on different cations contributes. It fluctuates mainly because of relative translational motions of the $\mathrm{Pe}_{4} \mathrm{~N}^{+}$cations. A large mean distance between the cations and slow motions render this relaxation path of minor importance, however. It may be calculated within a diffusion model with reflecting boundary conditions at the distance of closest approach $d_{\mathrm{HH}}$ of the cations but which neglects any structure in the radial pair distribution function $g_{\mathrm{HH}}(r)$. The corresponding relaxation rate is then given by $^{27}$

$R_{1, \mathrm{POS}}(\mathrm{H}, \mathrm{H})=$

$$
(6 \pi / 5)\left(\gamma_{\mathrm{H}} \gamma_{\mathrm{H}} \hbar \mu_{0} / 4 \pi\right)^{2}\left(g_{\mathrm{POS}}\left(\omega_{\mathrm{H}}\right)+4 g_{\mathrm{POS}}\left(2 \pi_{\mathrm{H}}\right)\right)
$$

The spectral density function for diffusive positional fluctuations is given by

$$
\begin{aligned}
& g_{\mathrm{POS}}\left(\omega_{\mathrm{H}}\right)=\left(N_{\mathrm{s}} \tau_{\mathrm{pos}} /\left(d_{\mathrm{HH}}\right)^{3}\right)\left[\left((3 / 2) u^{2}+(15 / 2) u+\right.\right. \\
& 12) /\left((1 / 8) u^{6}+u^{5}+4 u^{4}+(27 / 2) u^{3}+(81 / 2) u^{2}+\right.
\end{aligned}
$$$$
81 u+81)]
$$

with $N_{\mathrm{S}}$ the number density of the protons which can be calculated from the known mass density. ${ }^{11}$ The relations

$$
u^{2}=2 \omega_{\mathrm{H}} \tau_{\mathrm{POS}} \quad \text { and } \quad \tau_{\mathrm{pos}}=\left(d_{\mathrm{HH}}\right)^{2} / D_{\text {rel }}(\mathrm{H}, \mathrm{H})
$$

define the correlation time $\tau_{\mathrm{POS}}$ for translational motions of the cations. The relative translational diffusion coefficient $D_{\text {rel }}(\mathrm{H}, \mathrm{H})$ will be approximated by

$$
D_{\text {rel }}(\mathrm{H}, \mathrm{H})=2 D^{+}(\mathrm{H})
$$

with $D^{+}(\mathrm{H})$ the experimental self-diffusion coefficient of the protons on the cations. The distance of closest approach $d_{\mathrm{HH}}$ is taken to correspond to the radius of the $\mathrm{Pe}_{4} \mathrm{~N}^{+}$cations for consistency, thus $d_{\mathrm{HH}}=0.529 \mathrm{~nm}$. It is assumed to be independent of temperature so that $\tau_{\text {pos }}(T)$ exhibits the same temperature dependence as the experimental self-diffusion coefficient. The latter is much stronger than the modest variation of the spin density $N_{\mathrm{S}}$ with temperature, which, furthermore, is unknown and has been ignored. Anyhow, the contribution of $R_{1, \text { POS }}$ to the total proton relaxation rate $R_{1}\left({ }^{1} \mathrm{H}\right)$ is small in all cases considered.

The intramolecular contribution $R_{1, \mathrm{OR}}$ to the proton relaxation rate is related to the dipolar coupling of all protons located on the same cation. These interactions fluctuate due to overall tumbling of the whole cation as well as to segmental motions of the aliphatic chains. The corresponding relaxation rate between a nuclear spin $I$ and $n$ equivalent spins $I^{\prime}$ is given in general by ${ }^{16}$

$$
R_{1}(I, I)=n(2 / 5)\left(\gamma_{\mathrm{H}} \gamma_{\mathrm{H}} \hbar \mu_{0} / 4 \pi\right)^{2} I^{\prime}\left(I^{\prime}+1\right)\left\{g\left(\omega_{\mathrm{H}}\right)+4 g\left(2 \omega_{\mathrm{H}}\right)\right\}
$$

With overall tumbling motions and internal rotations taken to be uncoupled, the correlation function $G(t)$ is obtained as given in eq 8 with $R_{\mathrm{CH}}$ replaced by $R_{\mathrm{HH}}=0.178 \mathrm{~nm}$, the proton-proton distance within an alkyl group. The corresponding spectral density function $g_{O R}(\omega)$ is then given by eq 9 .

Due to the $R^{-6}$ dependence on internuclear separation, the dipolar coupling within an alkyl group dominates the relaxation rate in all cases except the $\mathrm{C}_{\alpha} \mathrm{H}_{2}$ and $\mathrm{C}_{\beta} \mathrm{H}_{2}$ groups close to the nitrogen core of the cations. The protons of the $\alpha$-methylene group on one aliphatic chain may interact strongly with the protons of the $\alpha$ - and $\beta$-methylene groups at other aliphatic chains of the cation in certain conformations. In case of an all-trans conformation of all four aliphatic side chains the dipolar contributions have been summed up for every alkyl chain. The corresponding factors

$$
Q_{i}=\left[\Sigma\left(1 / R_{\mathrm{HH}^{\prime}}\right)^{6}\right] /\left(1 / R_{\mathrm{HH}^{\prime}}\right)^{6}, \quad i=\alpha, \ldots, \epsilon
$$

are given in Table $V$." With the presumably small effect on relaxation of the modulation through segmental motions of the internuclear distances of spins on different alkyl groups neglected, ${ }^{28}$ eq 8 has been multiplied by the factor corresponding to the alkyl group under consideration to evaluate the related experimental relaxation rate $R_{1, \mathrm{OR}}\left({ }^{1} \mathrm{H}_{n}\right)$. The latter exhibits a temperature dependence identical to the corresponding ${ }^{13} \mathrm{C}$-relaxation rates $R_{1, O R}\left({ }^{13} C_{n}\right)$, hence the apparent activation energy $E_{\mathrm{a}}$ for the correlation time of the segmental motion $\tau_{n}$, has been kept constant. The generalized order parameter $\left(S_{n}\left({ }^{\prime} \mathrm{H}\right)\right)^{2}$ of the segmental motion must, however, be expected to differ from the one relevant to ${ }^{13} \mathrm{C}$ relaxation because of different orientations of the relevant principal axis systems relative to the diffusion frame centered at the nitrogen core. They have been adjusted to fit the experimental rates and are given in Table $\mathrm{V}$ also. These order parameters consistently reflect the same trend as those deduced from ${ }^{13} \mathrm{C} R_{1}$. With increasing distance from the highly mobile terminal methyl group toward the nitrogen center, the order parameter increases considerably. This trend clearly demonstrates the increasing importance of overall tumbling motions modulating the dipolar coupling of the protons of a given methylene group and causing an efficient spin relaxation. The $\alpha$-methylene group proton relaxation is mainly dominated by this mode, though not totally as suggested in the literature. ${ }^{16}$ This is further corroborated by the similar temperature dependence of the $\alpha$-methylene group ${ }^{1} \mathrm{H}$ and ${ }^{13} \mathrm{C}$ relaxation rates and the cationic diffusivity, which is distinctly different from the temperature dependence of the corresponding methyl group relaxation rates. Hence it is difficult to obtain direct dynamic information about the overall tumbling mode from proton as well as carbon-13 relaxation data.

It has also been suggested that relaxation of the ${ }^{14} \mathrm{~N}$ nuclear spin system might be an alternative. Due to the electric quadrupole moment of the nitrogen-14 nucleus, the relaxation is dominated by the coupling of the electric quadrupole moment to the electric field gradient at the nuclear site. The latter originates from the surrounding charge distribution and is highly quenched due to the high symmetry of the $\mathrm{Pe}_{4} \mathrm{~N}^{+}$ion. Fluctuations of this coupling causing relaxation arise thus mainly from molecular motions of the surrounding solvent dipoles and anions hence provide no direct information about reorientational motions of the $\mathrm{Pe}_{4} \mathrm{~N}^{+}$ions. Figure 7 shows the ${ }^{14} \mathrm{~N}$ relaxation times of the solute and the solvent molecules as a function of the concentration. Though 


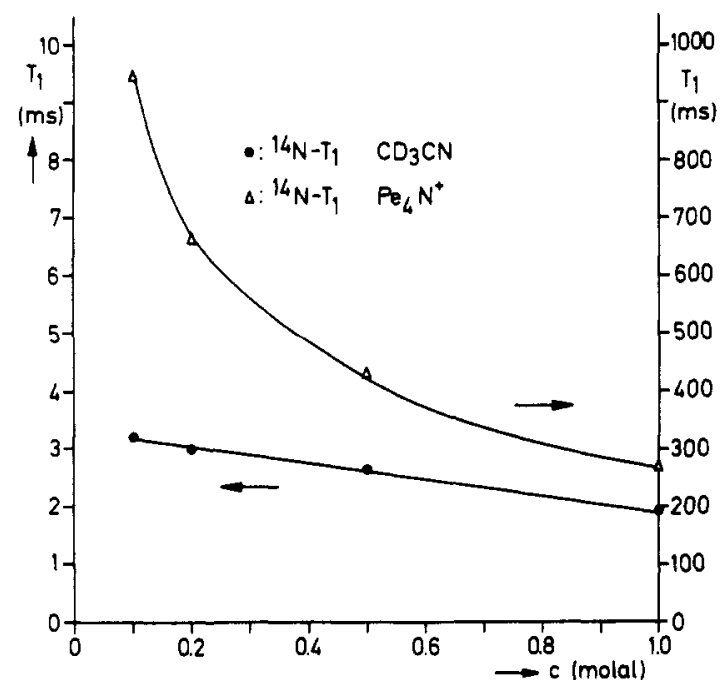

Figure 7. Isothermal $(T=295 \mathrm{~K})$ concentration dependence of the nitrogen-14 spin-lattice relaxation rate of the solute $\mathrm{Pe}_{4} \mathrm{~N}^{+}$cations and the solvent $\mathrm{CD}_{3} \mathrm{CN}$ molecules.

largely unrelated at low solute concentration, both relaxation times show an increasingly similar behavior at high concentrations.

\section{Conclusions}

Dynamic information about $\mathrm{Pe}_{4} \mathrm{~N}^{+}$ions dissolved in $\mathrm{CD}_{3} \mathrm{CN}$ has been gathered with NMR. Self-diffusion coefficients and proton and carbon-13 spin-lattice relaxation rates have been determined over a large concentration and temperature range. These transport coefficients show a simple Arrhenius temperature dependence, indicating that significant ion pairing is unimportant in the whole temperature range. From chemical shift measurements it has been concluded that ion pairing would polarize the pasitive charge density which is centered at the nitrogen core and further smeared out over the neighboring $\alpha$-methylene groups. This suggests that ion pairing would also affect the relaxation behavior of the $\alpha$-methylene protons and carbon-13 nuclei. This has not been observed, however. Thus acetonitrile effectively solvates $\mathrm{Pe}_{4} \mathrm{~N}^{+}$ions in the whole range of concentrations and temperatures.

The relaxation rate maximum detected in the $1 \mathrm{~m}$ solution clearly demonstrates the strong slowing down of the whole-ion reorientations in this system. The observation that the $\alpha$-methylene group proton and carbon-13 relaxation is dominated by the overall tumbling mode shows that the segmental mobility of these groups is strongly reduced. Hence these local motions of the alkyl groups exhibit correlation times similar to those for whole-ion tumbling motions. A large spatial restriction of these segmental motions is indicated by order parameters $S_{n}$ close to 1 and is consistent with these conclusions also.

Self-diffusion coefficients of the organic ions have been found to differ from those deduced from QENS data. It is argued that the difference arises from the contribution of a hydrodynamic and a relaxational mode to the diffusion process. Only the former is detected by QENS experiments because of the shorter time scale involved, whereas both contributions are detected by the slower NMR method. It is further argued that this effect may only be observed in low-viscosity liquids.

Acknowledgment. Two of the authors (E.W.L. and S.B.) thank the DFG for supporting this work. The skillful technical help of S. Heyn, R. Knott, and E. Treml made this work feasible.

Registry No. $\mathrm{Pe}_{4} \mathrm{NBr}, 866-97-7 ; \mathrm{Pe}_{4} \mathrm{~N}^{+}, 15959-61-2$; acetonitrile, 75-05-8

\title{
Translational Energy Dependence of Gas-Phase Reactlons of Halides with Halogenated
}

\section{Alkanes}

\author{
C. E. C. A. Hop and T. B. McMahon*
}

Department of Chemistry and Guelph-Waterloo Centre for Graduate Work in Chemistry, University of Waterloo, Waterloo, Ontario, N2L 3G1 Canada (Received: June 24, 1991)

\begin{abstract}
The gas-phase bimolecular nucleophilic substitution reactions $\mathrm{Br}^{-}+\mathrm{CCl}_{4} \rightarrow \mathrm{BrCCl}_{3}+\mathrm{Cl}^{-}, \mathrm{Br}^{-}+\mathrm{CF}_{2} \mathrm{Cl}_{2} \rightarrow \mathrm{BrCF}_{2} \mathrm{Cl}+$ $\mathrm{Cl}^{-}$, and $\mathrm{Cl}^{-}+\mathrm{CBr}_{4} \rightarrow \mathrm{ClCBr}_{3}+\mathrm{Br}^{-}$were studied as a function of the center-of-mass energy with Fourier transform ion cyclotron resonance spectrometry. From the energy dependence and the threshold energies of these reactions, conclusions were drawn concerning the mechanism involved.
\end{abstract}

\section{Introduction}

Bimolecular nucleophilic displacement reactions $\left(\mathrm{S}_{\mathrm{N}} 2\right)$ reactions have played a fundamental role in the development of mechanistic organic chemistry. These reactions have been studied theoretically ${ }^{1}$ and experimentally, ${ }^{2-13}$ both in the gas phase ${ }^{2-8}$ and in solution, ${ }^{-13}$

(1) (a) DeTar, D. F.; McMullen, D. F.; Luthra, N. P. J. Am. Chem. Soc. 1978, 100, 2484. (b) Carrion, F.; Dewar, M. J. S. J. Am. Chem. Soc. 1984 106, 3531. (c) Černušăk, I.; Urban, M. Collect. Czech. Chem. Commun. 1988, 53, 2239. (d) Tucker, S. C.; Truhlar, D. G. J. Phys. Chem. 1989, 93 , 8138. (e) Vetter, R.; Zülicke, L. J. Am. Chem. Soc. 1990, 1l2, 5136 and references therein. (f) Shi, Z.; Boyd, R. J. J. Am. Chem. Soc. 1990, 112, 6789.

(g) Shi, Z.; Boyd, R. J. J. Am. Chem. Soc. 1991, II3, 1072. and the "back side attack" mechanism has become widely accepted. ${ }^{2}$ The potential energy surface for gas-phase $S_{N} 2$ reactions

(2) Riveros, J. M.; José, S. M.; Takashima, K. Adv. Phys. Org. Chem. 1985, 21, 197 and references therein.

(3) (a) Bohme, D. K.; Young, L. B. J. Am. Chem. Soc. 1970, 92, 7354 (b) Young, L. B.; Lee-Ruff, E.; Hohme, D. K. J. Chem. Soc., Chem. Commun. 1973, 35. (c) Bohme, D. K.; Mackay, G. I.; Payzant, J. D. J. Am. Chem Soc. 1974, 96, 4027. (d) Tanaka, K.; Mackay, G. I.; Payzant, J. D.; Bohme, D. K. Can. J. Chem. 1976, 54, 1643. (e) Bohme, D. K.; Raksit, A. B. J. Am Chem. Soc. 1984, 106, 3447. (f) Bohme, D. K. In Ionic Processes in the Gas Phase; Almoster Ferreira, M. A., Ed.; D. Reidel Publishing Company: Dordrecht, 1984: pp 111-134.

(4) Barlow, S. E.; Van Doren, J. M.; Bierbaum, V. M. J. Am. Chem. Soc. $1988,110,7240$. 